A trial fibrillation (AF) is the most commonly encountered arrhythmia in clinical practice. ${ }^{1}$ Recent advances in technology and in the understanding of the pathophysiology of AF have led to more definitive and potentially curative therapeutic approaches. ${ }^{1}$ In this setting, echocardiography has a unique and important role in the assessment of cardiac structure and function, risk stratification, and increasingly in guiding the management of AF. Because of its recognised value, echocardiography has become established in guidelines for management of $\mathrm{AF}^{2}$ and utilisation of echocardiography has increased, particularly of transoesophageal echocardiography to guide direct current cardioversion or detect cardiac sources of embolism. Even more recently the development of intracardiac echocardiography has led to real-time guidance of percutaneous interventions, including radiofrequency ablation and left atrial appendage closure procedures for patients with AF.

In this review, we highlight the echocardiographic modalities that are available and their role in the evaluation and management of AF.

\title{
ATRIAL FIBRILLATION
}

AF affects approximately $0.4 \%$ of the general population and its prevalence is increasing. ${ }^{34} \mathrm{AF}$ frequently accompanies common conditions such as hypertension, chronic heart failure, and valvar or ischaemic heart disease, and is an important sequela of cardiothoracic surgery. ${ }^{5}$ Importantly, $\mathrm{AF}$ is associated with significant mortality and morbidity, particularly from thromboembolic stroke. ${ }^{3-9}$ The risk of stroke is greater in the elderly and with concomitant valvar (particularly rheumatic) heart disease; however, non-valvar AF is responsible for 75000 strokes and hospitalisation costs of $\$ 1$ billion dollars annually in the USA. ${ }^{10}$ In addition, AF may be associated with reduced functional capacity and impaired cardiac performance, particularly when ventricular rates are not adequately controlled.

Despite a mounting disease burden, there have been significant advances in the management of AF because of a greater mechanistic understanding of pathophysiology. ${ }^{1}$ The aetiology of AF is complex and may be modulated by factors such as autonomic tone, atrial wall stress, inflammation, and ischaemia. The arrhythmia is characterised by disorganised electrical activity with multiple re-entrant circuits in the atria that can lead to electrical and structural remodelling of the atria, which in turn reinforces the establishment of AF. Once changes in atrial structure and mechanical function become gross, they can be detected by echocardiography.

Perhaps most significant is the discovery that a proportion of AF cases caused by triggering from foci within the pulmonary veins can be successfully ablated with radiofrequency treatment. Additionally, the left atrial appendage (LAA) has become a focus of attention with surgical and percutaneous closure techniques becoming available. Echocardiography has an increasingly important therapeutic role in guiding these ablation and LAA closure procedures.

\section{ECHOCARDIOGRAPHIC MODALITIES}

\section{Transthoracic echocardiography}

Transthoracic echocardiography (TTE), including two dimensional (2D) imaging and complete Doppler assessment of valves, is recommended for all subjects with AF. ${ }^{2}$ TTE allows rapid, safe,

See end of article for authors' affiliations

Correspondence to:

Allan L Klein MD, Cleveland Clinic Foundation, Department of Cardiovascular Medicine, 9500 Euclid Avenue, Desk F15, Cleveland, $\mathrm{OH} 44195$, USA; kleina@ccf.org relatively comprehensive assessment of cardiac structure and function that can help to define the underlying aetiology of AF and the risk of complications. Recent advances such as harmonic imaging, alone or with micro-bubble contrast agents, allows enhanced endocardial border definition for assessment of left ventricular volumes and function. New modalities such as colour M mode (CMM) and tissue Doppler imaging (TDI) allow more accurate assessment of diastolic function and estimated filling pressures. Assessment of systolic and diastolic left ventricular function in AF may, however, be complicated by irregular RR interval and rapid ventricular rate. Transthoracic imaging usually provides suboptimal visualisation of the atrial appendages and has inadequate sensitivity and specificity for diagnosing LAA thrombus. ${ }^{11}$ However, efforts continue 
to overcome this deficiency, including the use of microbubble contrast agents for better visualisation.

\section{Transoesophageal echocardiography}

Transoesophageal echocardiography (TOE), performed with

3-7 MHz multiplane transducers mounted on steerable modified fibroscopy probes, allows high resolution interrogation of posterior cardiac structures, including the atria, interatrial septum, and pulmonary veins. Most importantly, the atrial appendages can be visualised for thrombus. More accurate evaluation of valvar lesions, especially prosthetic dysfunction, is possible by TOE and alternative thromboembolic sources can be readily identified, including complex atheroma of the ascending thoracic aorta and arch. Although TOE is very safe, with major complications occurring in $<0.02 \%$ of procedures, it is not indicated for all patients in $\mathrm{AF}$, but generally performed in patients at high risk of thromboembolism, those being considered for early cardioversion, or those with a secondary indication such as valvar disease. $^{2}$

\section{Intracardiac echocardiography}

Intracardiac echocardiography (ICE) is performed with a 6$12 \mathrm{MHz}$ transducer placed in the right atrium via a 6-10 French sheath in the right femoral vein. ${ }^{12}$ Modified from intravascular ultrasound probes, these steerable monoplane transducers produce ultrasound waves mechanically or, like TOE, via a phased array. The latter offer the same modalities as TOE including colour, pulsed wave, and continuous wave Doppler, to allow assessment of intracardiac flow in addition to $2 \mathrm{D}$ visualisation of structures. ${ }^{12}$

ICE can play a key role in the guidance of cardiac interventions and in some centres is used routinely for radiofrequency ablation procedures as well as recently for percutaneous closure of patent foramen ovale or atrial septal defects. The utility and cost effectiveness of ICE in these settings is still being evaluated.

Table 1 summarises the indices measured by these different echocardiographic modalities.

Table 1 Summary of the indices measured by different echocardiographic modalities

Transthoracic echo

LA dimensions and volumes

LV dimensions and volumes

LVEF

LV diastolic function

Valvar function

Transoesophageal LA/LAA: structure, function, SEC, and thrombus

echo RA/RAA structure, function and thrombus Pulmonary vein anatomy/flows Inter-atrial septum (PFO, etc) Ascending aorta and arch atheroma Valvar function Guide therapy*

Intracardiac echo

LA/LAA structure and function LA/LAA thrombus Inter-atrial septum Pulmonary vein anatomy/flows Guide therapy*

*Radiofrequency ablation, valvoplasty, PFO/ASD closure devices, LAA occlusion device insertion.

ASD, atrial septal defect; LA, left atrium; LAA, left atrial appendage; LV, left ventricle; LVEF, left ventricular ejection fraction; PFO, patent foramen ovale; RA, right atrium; RAA, right atrial appendage; SEC, spontaneous echo contrast.

\section{ECHOCARDIOGRAPHIC ASSESSMENT OF AF AETIOLOGY}

Transthoracic echocardiography readily identifies important underlying conditions that predispose to $\mathrm{AF}$, including left ventricular systolic dysfunction caused by ischaemic or dilated cardiomyopathy, left ventricular hypertrophy caused by hypertension, valvar heart disease-particularly rheumatic mitral stenosis—or pericardial disease.

A normal heart (no structural abnormality) at echocardiography is significant, particularly in young subjects, as it suggests a triggered mechanism for AF that may be amenable to radiofrequency ablation.

\section{ECHOCARDIOGRAPHIC ASSESSMENT OF CARDIAC STRUCTURE AND FUNCTION IN AF}

A thorough TTE should include quantification of chamber dimensions, wall thickness, left ventricular systolic and diastolic function, and valvar function. Indices such as left atrial size, left ventricular wall thickness, and left ventricular dysfunction are independent predictors for the development of AF. For assessment of diastolic function, abnormal myocardial relaxation is detectable by reduced early diastolic mitral annular (Ea) velocities from TDI or by a delayed velocity of early left ventricular flow propagation ( $\mathrm{Vp}$ ) from CMM. The ratio of peak early transmitral velocity (E) to early diastolic annular (Ea) velocity (the E/Ea ratio) has been shown to reliably estimate left ventricular filling pressures in subjects in AF. ${ }^{13}$

The anteroposterior left atrial dimension is measured in the parasternal long or short axis from $\mathrm{M}$ mode or 2D imaging, while left atrial area or volume (modified Simpson's rule) can be measured in the apical two and four chamber views. The atria are commonly enlarged in AF and continue to dilate with persistent $\mathrm{AF}$, but may diminish in size when sinus rhythm is restored. Increased left atrial volume may be a stronger predictor of incident AF than left atrial dimensions alone, while severe left atrial dilatation is associated with a low probability of successful cardioversion for chronic AF. Left atrial mechanical function is difficult to evaluate during $\mathrm{AF}$, but when sinus rhythm is restored, it can be assessed with the transmitral atrial (A) wave velocity from pulsed wave Doppler and the mitral annular late diastolic (Aa) velocity from TDI. Depending on AF duration, the peak transmitral A wave velocity may be reduced for up to four weeks after successful cardioversion, due to atrial stunning.

TOE is the modality of choice for assessing the LAA and detecting left atrial and LAA thrombi (fig 1A). Modern multiplane TOE in experienced hands can detect thrombi with a sensitivity and specificity in the order of $95-100 \%$. TOE should be used to confirm thrombus resolution before subsequent cardioversion in patients with documented thrombus. Up to $80 \%$ of left atrial thrombi have disappeared after seven weeks of anticoagulation. TOE also accurately identifies spontaneous echo contrast (SEC or "smoke") and "sludge". LAA smoke (fig 1B), identified as swirling echodensity in the LAA and left atrium, is a marker of stasis and a probable precursor of thrombus. Severe SEC or smoke is associated with later development of LAA thrombus and with systemic embolisation. ${ }^{13}{ }^{14}$ LAA sludge (fig lC) is viscid but not solid echodensity in the LAA without clear thrombus formation. While the distinction is arbitrary and subjective, sludge represents a stage further along the continuum towards thrombus formation and appears to have greater prognostic significance than smoke. LAA mechanical function, 

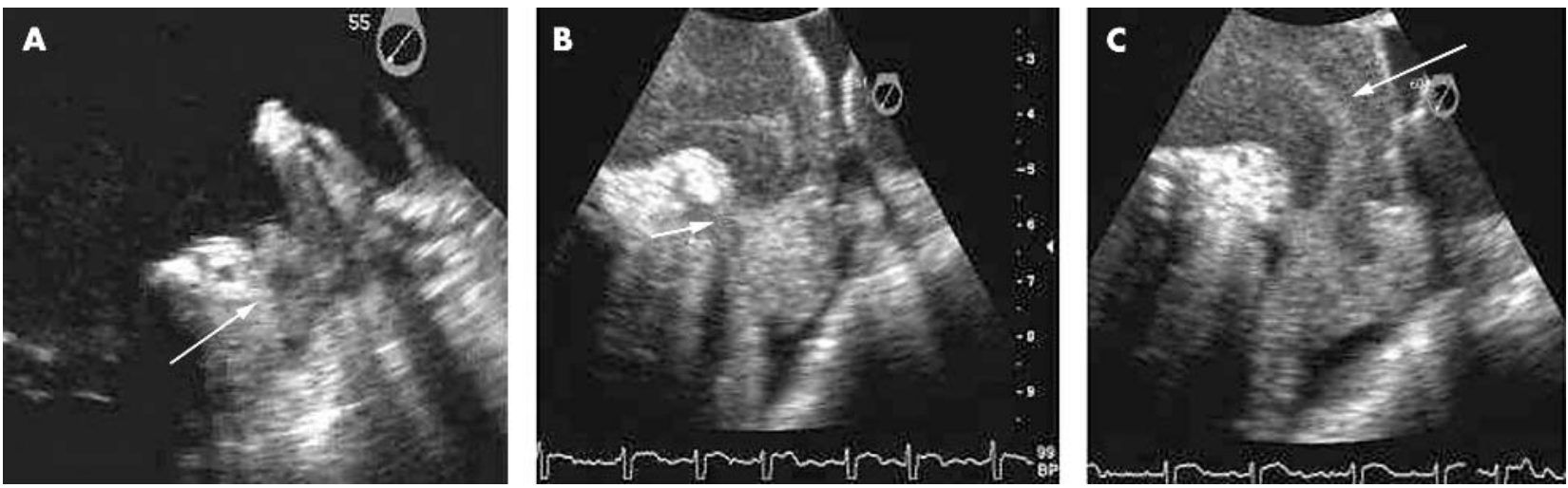

Figure 1 Images of the left atrium and left atrial appendage (LAA) depicting the spectrum of flow stasis and thromboembolic risk from "smoke" through thrombus. (A) LAA thrombus detected in a patient with atrial fibrillation (AF) undergoing transoesophageal echo (TOE) before cardioversion. Note the well formed rounded upper edge of the thrombus (arrow). (B) "Sludge" in the apex of the LAA detected by TOE in a patient with AF. Note the meniscus at the upper edge of the sludge (arrow). (C) Spontaneous echo contrast or "smoke" swirling detected by TOE in the LAA and left atrium of a patient in AF.

including LAA emptying velocities, can be assessed from TOE using pulsed wave Doppler with a sample volume placed $1 \mathrm{~cm}$ into the appendage at transducer phased array angles between $45-120^{\circ}$. Low LAA velocities $(<20 \mathrm{~cm} / \mathrm{s})$ correlate strongly with the presence of spontaneous echo contrast and thrombus formation, ${ }^{15}{ }^{16}$ while LAA emptying velocities $>40 \mathrm{~cm} / \mathrm{s}$ predict a greater likelihood of sustained sinus rhythm one year after cardioversion. ${ }^{17}$ The concept of LAA stunning, particularly following direct current (DC) cardioversion, is important. TOE performed immediately before and after DC cardioversion demonstrates a decline in left atrial and LAA function and increased smoke in a significant number of patients. New LAA thrombus formation has been demonstrated immediately after DC cardioversion. Stunning has been seen with spontaneous and pharmacological cardioversion as well. ${ }^{14}$ The severity and duration of atrial stunning appears to reflect the duration of antecedent AF.

TOE also allows more accurate assessment of pulmonary vein systolic, diastolic, and atrial reversal (in sinus rhythm) flow velocities, which is particularly relevant in patients undergoing pulmonary vein isolation procedures.

Finally, TOE allows accurate diagnosis of important associated lesions that may cause thromboembolism, in particular, complex ascending aortic and arch atheroma.

\section{ECHOCARDIOGRAPHIC ASSESSMENT OF COMPLICATIONS AND MORTALITY IN AF}

$\mathrm{AF}$ is an independent predictor of increased mortality in the general population. ${ }^{8}$ Among subjects with AF, several echocardiographic indices predict increased mortality, including presence of thrombus and left ventricular systolic dysfunction. Clinical factors such as age, prior thromboembolism, hypertension, diabetes or heart failure are strong independent predictors of stroke in AF. Several echocardiographic indices also predict thromboembolic stroke, ${ }^{15}$ including rheumatic mitral valve disease, left ventricular systolic dysfunction or hypertrophy on TTE, and markers of left atrial/ LAA dysfunction or stasis on TOE. ${ }^{16}$ In the SPAF (stroke prevention in atrial fibrillation) III study the presence of a left atrial abnormality (LAA thrombi, LAA flow velocities $<20 \mathrm{~cm} / \mathrm{sec}$, or severe spontaneous echo contrast) or complex aortic atheroma at TOE was associated with a higher risk of thromboembolism. Patients with both left atrial abnormality and complex aortic atheroma had an annual incidence of embolic events in the order of $21 \%$ compared to $1.4 \%$ in the absence of these findings. In other series, the presence of a left atrial thrombus on TOE is associated with an annual risk of stroke in excess of $10 \%$ and an annual rise of death in excess of $15 \% .{ }^{17}$ Hence, TTE and TOE findings may provide prognostic information that is additive to traditional clinical factors. ${ }^{16}$

\section{ECHOCARDIOGRAPHIC GUIDANCE OF TREATMENT IN AF}

\section{TOE guided cardioversion}

DC cardioversion for AF offers the opportunity to restore sinus rhythm, optimise cardiac performance, and potentially reduce the risk of thromboembolic events and bleeding complications caused by prolonged anticoagulation. ${ }^{14}$ To avoid thromboembolic events ( $\sim 5 \%$ in non-anticoagulated patients) around the time of cardioversion, patients with AF of more than 48 hours' duration have traditionally been anticoagulated for at least seven weeks-three weeks before and four weeks after the procedure. With this approach, thromboembolic complications $(<1 \%)$ and major bleeding $(1-2 \%)$ are infrequent. The procedure is initially successful in up to $86 \%$ of subjects with sinus rhythm maintained in up to $50 \%$ at one year. Predictors of successful cardioversion include age, duration of $\mathrm{AF}$, underlying aetiology, and technical factors. Additionally, severe left atrial dilatation and left ventricular systolic dysfunction on echocardiography predict a lower likelihood of success. ${ }^{14}$

\section{Echocardiographic predictors of} thromboembolism in AF

- Valve disease

- Left ventricular systolic dysfunction

- Left atrial dilatation

- ${ }^{*}$ Complex aortic atheroma

- *Left atrial appendage thrombus $>$ sludge $>$ smoke

* Reduced LAA velocities $(<20 \mathrm{~cm} / \mathrm{s})$

*Identified at TOE 
While the long term value of DC cardioversion has been brought into question by the recent findings of the AFFIRM (atrial fibrillation follow-up investigation of rhythm management) study, ${ }^{18}$ it is clear that symptomatic patients and a proportion of those who are asymptomatic will continue to receive benefit from this treatment.

1450 For some time, TOE has been used before cardioversion in patients presenting with AF of more than 48 hours' duration. TOE potentially facilitates early cardioversion without prolonged anticoagulation in low risk patients needing semiurgent cardioversion or for whom the risk of anticoagulation is high. TOE also identifies high risk patients with thrombus in whom DC cardioversion should be postponed.

The routine use of TOE guided DC cardioversion with short term anticoagulation has potential advantages over the conventional approach; these include the possibility of reduced embolic events due to recognition of LAA thrombi at TOE, shorter period of anticoagulation with less bleeding complications, higher rates of conversion to and maintenance of sinus rhythm due to prompt cardioversion, and less opportunity for atrial remodelling or stunning. The TOE guided strategy appears to be economically equivalent to the conventional approach.

A TOE guided approach to DC cardioversion does not obviate the need for therapeutic anticoagulation. Patients receiving short term therapeutic anticoagulation, and in whom thrombus had been excluded by TOE, experience low rates of embolism $(<1 \%)$-comparable to rates in subjects receiving three weeks of anticoagulation-following DC cardioversion from AF. ${ }^{4}$ Thromboembolic events have been documented following cardioversion from AF among patients with absent or subtherapeutic anticoagulation despite a negative TOE for thrombus, possibly caused by tiny thrombi not seen at TOE or as a result of thrombus formation caused by LAA stunning following cardioversion. Therefore, all patients should be adequately anticoagulated during and after cardioversion even when there is no LAA thrombus or the duration of AF is brief. ${ }^{10-12}$
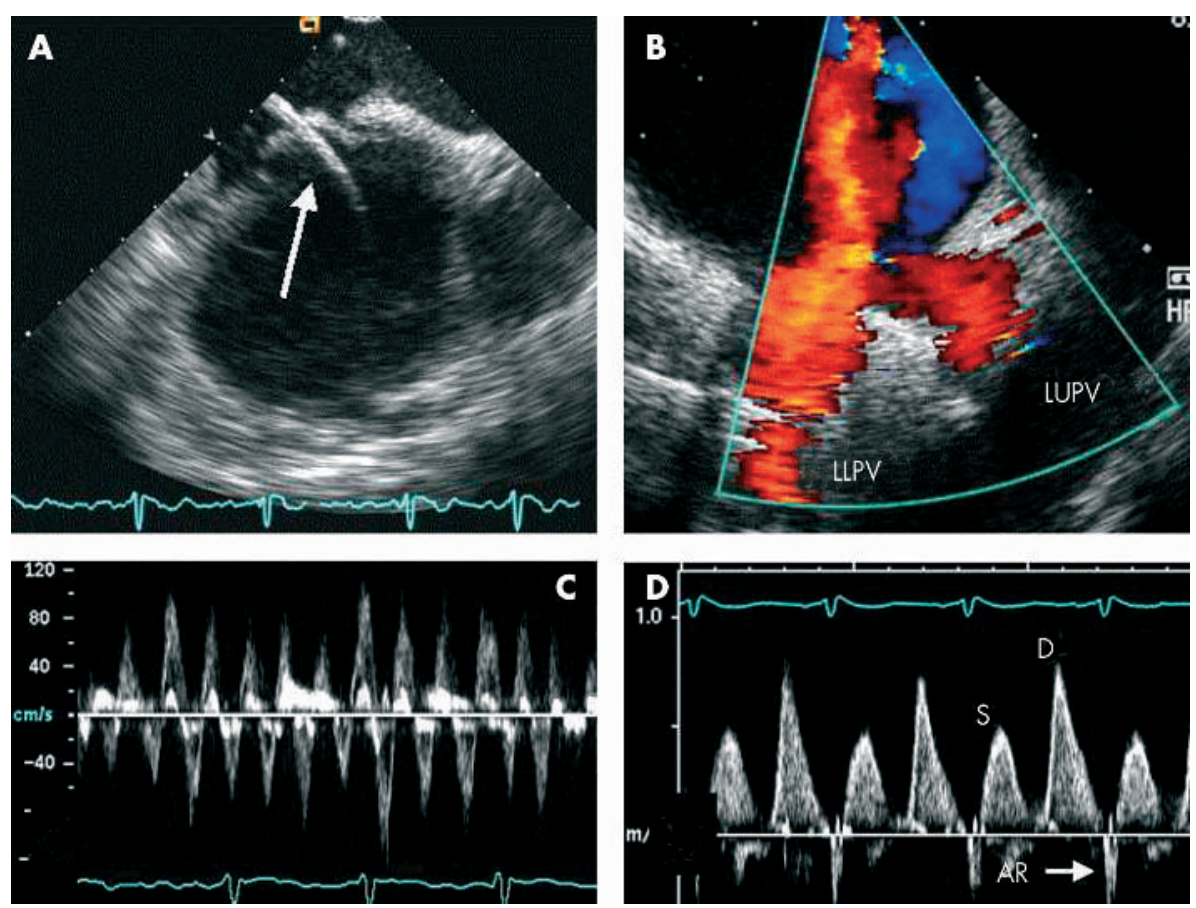

\section{Indications for echocardiography}

- Assessment of cardiac structure and function

- Assessment of aetiology of AF

- Assessment of thromboembolic risk or the aetiology of peripheral embolism

- TOE guided DC cardioversion/stratification for anticoagulation

- TOE guidance of percutaneous or surgical LAA closure

- ICE guided RF ablation procedures

The ACUTE (assessment of cardioversion using transoesophageal echocardiography) multi-centre study compared a TOE guided strategy with short term anticoagulation to a conventional anticoagulation strategy in patients undergoing DC cardioversion for AF of $>2$ days' duration. ${ }^{19}$ The primary end point events were ischaemic stroke, TIA, and systemic embolisation during an eight week period from enrolment. Six hundred and nineteen patients were randomised to the TOE guided arm with 603 assigned to the conventional arm. Of the 619 patients in the TOE guided group, 425 (69\%) had early DC cardioversion at a mean of three days; and in 344 $(81 \%)$ of these, the cardioversion was successful. Among the 124 patients who had TOE but not early DC cardioversion $(20 \%)$, cardioversion was postponed in 76 patients $(61 \%)$ due to the finding of thrombi. These 76 patients $(13.8 \%)$ were identified among the 549 who actually had the TOE exam. In the TOE guided group, there were five embolic events of which two patients experienced embolic events within the first week after cardioversion, associated with recurrence of $\mathrm{AF}$ and subtherapeutic anticoagulation.

Of the 603 in the conventional group, 333 (55\%) underwent DC cardioversion at a mean of 31 days; and in 266 $(80 \%)$ cardioversion was immediately successful. Of the 270 (45\%) who did not undergo cardioversion, $47 \%$ reverted to sinus rhythm spontaneously or with pharmacologic conversion. The remaining 53\% of patients did not undergo cardioversion within the eight week period due to patient
Figure 2 Images acquired from intracardiac echocardiography (ICE). (A) Transeptal puncture with catheter (arrow) passing from right atrium to left atrium. (B) Colour flow mapping showing the confluence of the left upper and lower pulmonary veins (LUPV and LLPV). (C) Pulsed wave Doppler of the LAA showing preservation of velocities in a patient with AF. (D) Pulsed Doppler of the LUPV showing blunting of systolic (S) flow compared to diastolic (D) flow and a narrow atrial systolic reversal (AR) following cardioversion in the same patient. 

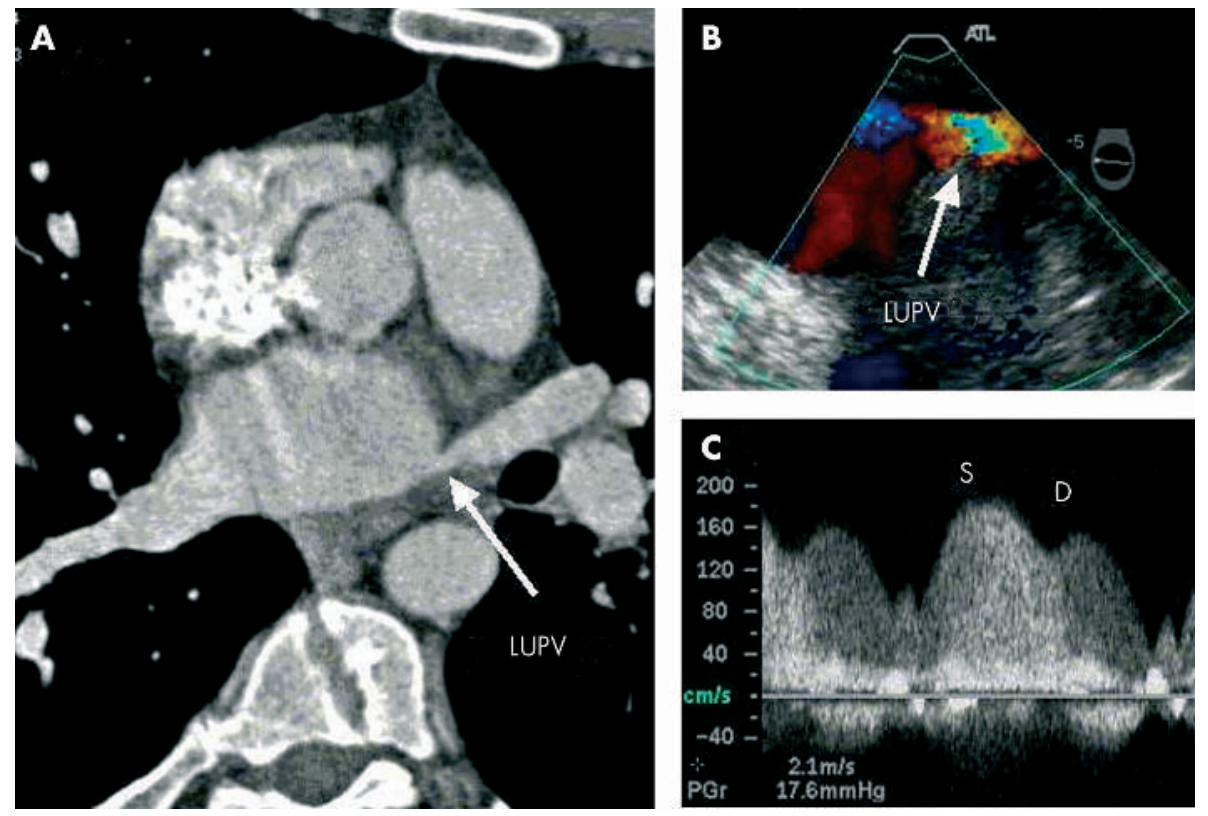

Figure 3 Stenosis of the left upper pulmonary vein (LUPV) following radiofrequency ablation for $\mathrm{AF}$. (A) Narrowing of the LUPV ostium (arrow) on multi-detector computed

tomography. (B) Colour Doppler image from TOE demonstrating aliasing of flow in the LUPV. (C) Pulsed wave Doppler of the LUPV demonstrating phasic systolic (S) and diastolic (D) flow with increased velocities $(>1.5 \mathrm{~m} / \mathrm{s})$

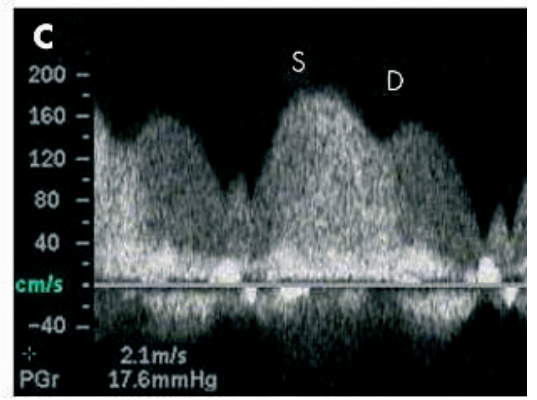

refusal, death, surgery, physician decision, subtherapeutic anticoagulation, bleeding or loss to follow up. In the conventional group, there were three embolic events of which one patient experienced an embolic event within the first week after cardioversion. Thirty two patients (5\%) crossed over to the TOE arm due to haemodynamic instability (hypotension or heart failure). There was no difference in the primary composite end point of stroke, TIA, and peripheral embolism between the TOE guided arm and conventional arms $(0.81 \% \vee 0.50 \% ; \mathrm{p}=0.50)$. However, the composite of major and minor bleeding occurred significantly less frequently in the TOE guided than the conventional arm $(3.1 \% \mathrm{v}$ $5.5 \% ; \mathrm{p}=0.023$ ). There was no significant difference between the two groups in the rate of death from cardiac causes and non-cardiac causes, but there was a trend for a higher rate of death in the TOE guided group $(2.4 \% \vee 1.0 \% ; \mathrm{p}=0.06)$.
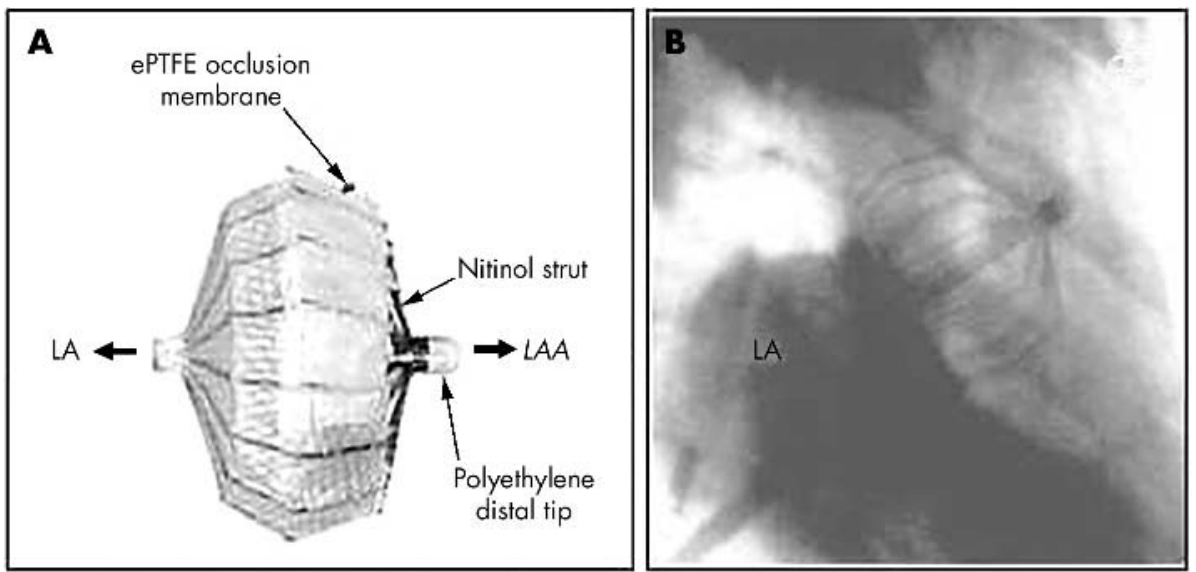

Figure 4 Percutaneous closure of the LAA. (A) Occluder device. (B) Fluoroscopic image of the occluder device in situ. (C) ICE image of the left atrium (LA) and LAA before deployment. (D) ICE following deployment of the device. Adapted and reproduced from Nakai et al ${ }_{1}^{20}$ with permission.
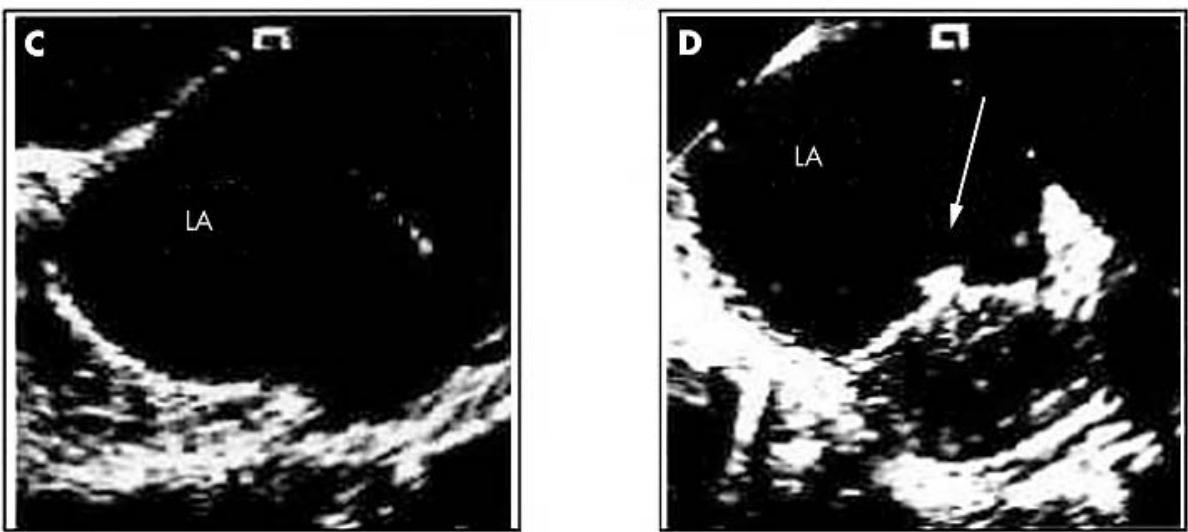
Recent onset $\mathrm{AF}$

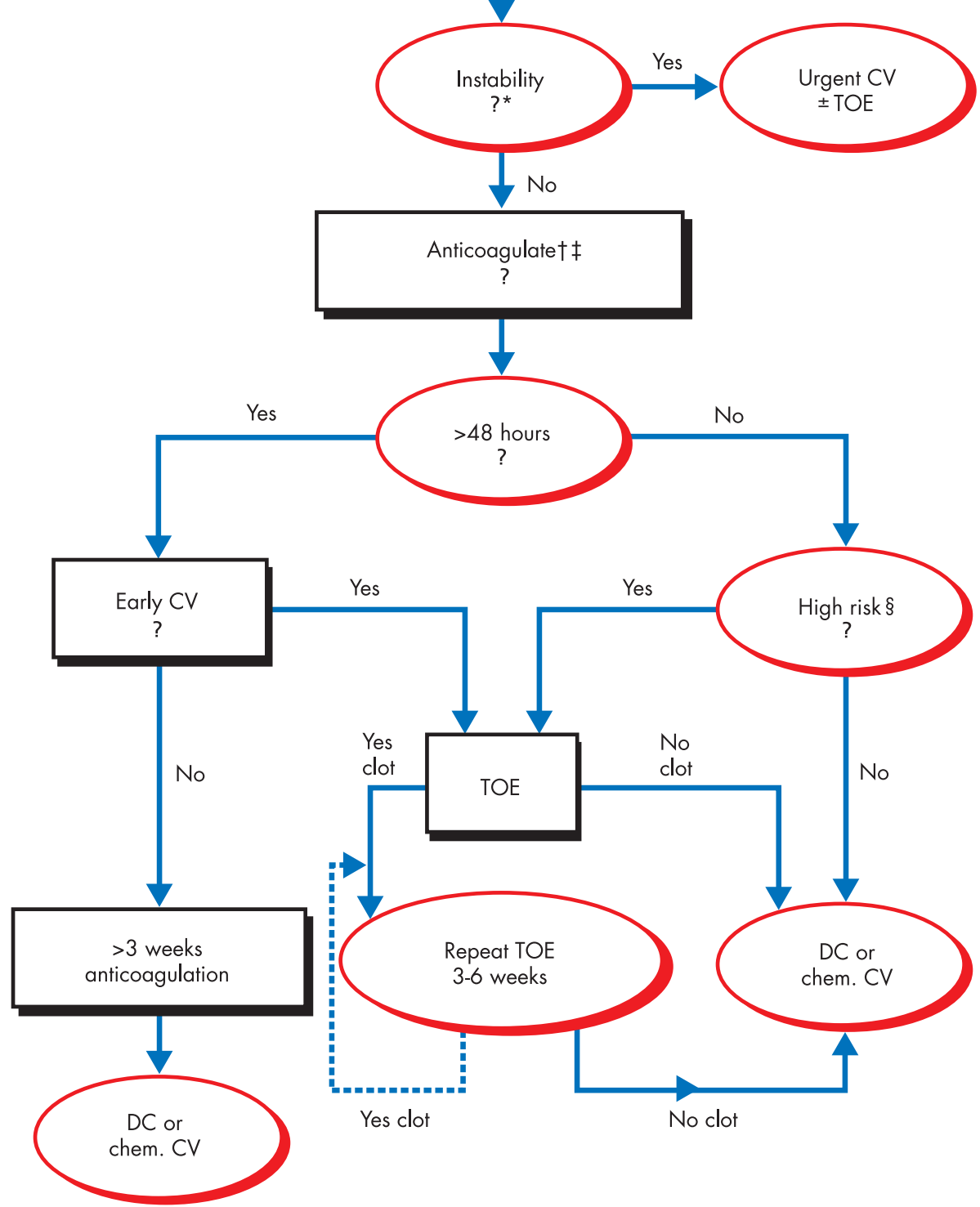

Figure 5 Proposed algorithm for the approach to patients presenting with recent onset atrial fibrillation. *Patients with haemodynamic instability, acute coronary syndrome, or severe heart failure. †If anticoagulation with heparin (unfractionated or low molecular weight) is contraindicated, consider transoesophageal echo guided cardioversion and review contraindication to anticoagulation if thrombus detected. \#If not high risk and $<48$ hours, no anticoagulation is needed. §High risk if recent left atrial appendage thrombus, prior thromboembolism, $>65$ years old, heart failure, hypertension, left ventricular dysfunction, significant valve disease, left atrial dilatation, increased risk of haemorrhagic complications, or suboptimal anticoagulation. $\mathrm{AF}$, atrial fibrillation; DC, direct current; Early CV, indication or preference for early cardioversion (see text); TOE transoesophageal echocardiogram.
Maintenance of sinus rhythm at eight week follow up was similar in the two groups $(53 \% \vee 50 \% ; \mathrm{p}=0.43)$.

In summary, the ACUTE study found that embolic events and maintenance of sinus rhythm were similar in the two treatment strategies, although major and minor bleeding was lower with the TOE guided strategy. These findings suggest that a TOE guided approach to DC cardioversion using short term anticoagulation is a safe and clinically effective alternative to the conventional strategy. The ACUTE II study will test whether low molecular weight heparin can be substituted for unfractionated heparin in this setting.

In patients presenting with a shorter duration of $\mathrm{AF}(<48$ hours) the risk of thromboembolism following cardioversion is low. While anticoagulation before cardioversion is recommended in these patients, TOE is not usually mandated unless patients are at high risk for thromboembolism. LAA thrombi may be present in up to $20 \%$ of patients presenting with new onset ( $<72$ hours) AF and a history of prior thromboembolism.

LAA dysfunction or stunning after cardioversion and LAA smoke or thrombus can be seen in atrial flutter. For this reason, a similar approach to cardioversion is generally taken for patients with atrial flutter.

In our practice (fig 5), we recommend TOE guided DC cardioversion for patients with new onset AF ( $>48$ hours) in three main circumstances ${ }^{19}$ : (1) for patients who require expedited (but not emergency) cardioversion because of significant symptoms, heart failure, angina, or haemodynamic instability; (2) patients with suboptimal anticoagulation or who cannot take anticoagulants because of a high risk of bleeding; and (3) patients at significant risk of left atrial/ LAA thrombus or stroke (see above). We also perform TOE 
guided DC cardioversion in a significant proportion of patients without these indications-particularly hospital inpatients-for whom expedited cardioversion is preferred.

\section{Radiofrequency ablation}

Radiofrequency ablation is suitable in a significant number of patients in whom AF is caused by foci in the pulmonary veins. ${ }^{1}$ Increasingly, ICE is being used to guide RF ablation procedures. ${ }^{12}$ Continuous imaging with ICE can be performed by the operator or an assistant and provides excellent visualisation of the atria and appendages, inter-atrial septum, and pulmonary veins. ICE facilitates trans-septal puncture, guide placement, and optimal tissue contact of mapping and ablation catheters, and allows identification of coagulum on the ablation catheter. ICE also allows detection of microbubbles at the ablation site, which may potentially guide optimal catheter placement and energy delivery. ${ }^{20}$ In addition, ICE facilitates instant recognition of significant complications such as pericardial effusion or tamponade, aortic puncture during trans-septal puncture, and may actually reduce the frequency of these complications. The use of ICE during radiofrequency ablation of the pulmonary vein ostia at our institution has been associated with shorter fluoroscopy and total procedure times, less energy use, higher success rates, and fewer complications including pulmonary vein stenosis (A Natale, personal communication). Figure 2 shows images acquired from ICE.

In the absence of ICE, TOE has an important role before ablation in assessing the left atrium and LAA for thrombus, the inter-atrial septum for patient foramen ovale (PFO), and for documenting baseline pulmonary vein flow profiles. TOE examination following catheter ablation of the pulmonary veins has documented asymptomatic increases in peak systolic flow velocities measured by Doppler in up to $40 \%$ of patients. Symptomatic pulmonary vein stenosis has been documented but is uncommon and may manifest as cough, dyspnoea, pulmonary hypertension, pulmonary oedema isolated to a single lobe, or with abnormal ventilation or perfusion lung scans in the absence of thromboembolism. ${ }^{21}$ With TOE, the pulmonary vein ostia can be visualised for evidence of stenosis; more importantly, the physiological significance of any narrowing can be assessed with colour flow mapping and pulsed or continuous wave Doppler of pulmonary vein flows (fig 3).

\section{Surgical procedures}

The surgical MAZE III procedure-involving excision or ligation of the appendages, isolation of the pulmonary veins and creation of incisions in the LA to reduce multiple re-entry circuits-abolishes AF in a significant number of patients. Due to the complexity of the operation, time on cardiopulmonary bypass, and bleeding risk, the latest evolution is to isolate the pulmonary veins and create lines of conduction block in the left atrium. This technique using heat based energy sources (radiofrequency) or cryotherapy is quicker and associated with fewer complications. TOE has an important role in assessing the atria and appendages for thrombus before the MAZE III procedure. Doppler echocardiography can also be used to track changes in atrial function or the presence of LAA communication with the left atrial cavity after the procedure.

Increasingly, LAA ligation is being undertaken at the time of cardiac surgery in patients at higher risk for AF or thromboembolism. Doppler imaging with intraoperative TOE allows immediate assessment of the efficacy of ligation at the time of surgery. We have seen a number of cases where the initial ligation was not adequate and communication between the LAA and left atrial cavity was detected.

\section{Percutaneous closure of the left atrial appendage}

Percutaneous occlusion of the left atrial appendage by an expandable occluder device is a new experimental approach (fig 4). ${ }^{22}$ Because $90 \%$ of thrombi occur in the LA appendage, occlusion of the LAA could potentially reduce the rate of thromboembolic complications in high risk patients or those who cannot be safely anticoagulated long term. In a canine model, insertion of the device was facilitated by ICE, which allowed optimal positioning of the device. Colour flow mapping was used to determine the optimal fit of the device and any significant residual flow out of the appendage (fig 4). ${ }^{22}$ TOE was used in a recent clinical study to guide placement and assess outcome and complications such as thrombus formation around the device. ${ }^{23}$

While further studies will be necessary to determine the role of this device in the treatment of AF, echocardiography is likely to play an important role in this validation process and is ideally suited to determining appropriate patients, guiding insertion of the device, and tracking potential complications.

\section{CONCLUSIONS}

Echocardiography provides vital information about cardiac function, complications, and prognosis in patients with AF. Modalities like TOE and ICE can help to guide ablation therapy and decisions related to cardioversion. The integrated use of echocardiography will be an important component in the optimal management of the looming AF epidemic.

\section{ACKNOWLEDGEMENTS}

The authors wish to acknowledge Marie Campbell for assistance in the preparation and review of the manuscript.

\section{$\cdots \cdots \cdots \cdots \cdots$}

Affiliations

R Troughton, Christchurch School of Medicine and Health Sciences, Christchurch, New Zealand

C R Asher, A L Klein, Cleveland Clinic Foundation, Department of Cardiovascular Medicine, Cleveland, Ohio, USA

Conflict of interest statement: Dr Klein has a research grant from Aventis. There are no financial or personal associations that may pose a conflict of interest in connection with the submitted article for any of the other authors to disclose.

\section{REFERENCES}

1 Peters NS, Schilling RJ, Kanagaratnam P, et al. Atrial fibrillation: strategies to control, combat, and cure. Lancet 2002;359:593-603.

- An excellent recent review of pathophysiology and current approaches to the management of atrial fibrillation.

2 Fuster V, Ryden LE, Asinger RW, et al. ACC/AHA/ESC guidelines for the management of patients with atrial fibrillation: executive summary. A report of the American College of Cardiology/American Heart Association task force on practice guidelines and the European Society of Cardiology committee for practice guidelines and policy conferences (committee to develop guidelines for the management of patients with atrial fibrillation) developed in collaboration with the North American Society of Pacing and Electrophysiology. Circulation 2001;104:2118-50.

- Recent policy guidelines for the management of AF produced after collaboration between the AHA, ACC, and ESC.

3 Kannel WB, Wolf PA, Benjamin EJ, et al. Prevalence, incidence, prognosis, and predisposing conditions for atrial fibrillation: population-based estimates. Am J Cardiol 1998:82:2N-9N.

4 Feinberg WM, Blackshear JL, Laupacis A, et al. Prevalence, age distribution, and gender of patients with atrial fibrillation. Analysis and implications. Arch Intern Med 1995;155:469-73. 
5 Ommen SR Odell JA Stanton MS Atrial arrhythmias after cardiothoracic surgery. N Engl J Med 1997;336:1429-34.

6 Kannel WB, Abbott RD, Savage DD, et al. Coronary heart disease and atria fibrillation: the Framingham study. Am Heart J 1983;106:389-96.

7 Dries DL, Exner DV, Gersh BJ, et al. Atrial fibrillation is associated with an increased risk for mortality and heart failure progression in patients with asymptomatic and symptomatic left ventricular systolic dysfunction: a retrospective analysis of the SOLVD trials. Studies of left ventricular dysfunction. J Am Coll Cardiol 1998;32:695-703.

Benjamin EJ, Wolf PA, D'Agostino RB, et al. Impact of atrial fibrillation on the risk of death: the Framingham heart study. Circulation 1998;98:946-52

- This paper provides important data on mortality in AF from a prospectively studied cohort.

9 SPAF Investigators. Warfarin versus aspirin for prevention of thromboembolism in atrial fibrillation: stroke prevention in atrial fibrillation II study. Lancet 1994;343:687-91.

10 Nattel S. Newer developments in the management of atrial fibrillation. Am Heart J 1995; 130:1094-106.

11 Omran H, Jung W, Rabahieh R, et al. Imaging of thrombi and assessment of left atrial appendage function: a prospective study comparing transthoracic and transoesophageal echocardiography. Hear 1999;81:192-8.

12 Cooper JM, Epstein LM. Use of intracardiac echocardiography to guide ablation of atrial fibrillation. Circulation 2001;104:3010-3

- An excellent review of this emerging echocardiographic modality and its role in interventional electrophysiology.

13 Nagueh SF, Kopelen HA, Quinones MA. Assessment of left ventricular filling pressures by Doppler in the presence of atrial fibrillation. Circulation 1996;94:2138-45

14 Klein AL, Murray RD, Grimm RA. Role of transesophageal echocardiographyguided cardioversion of patients with atrial fibrillation. J Am Coll Cardiol 2001;37:691-704

A comprehensive recent review of the role of TOE guided cardioversion for AF.
15 Anon. Risk factors for stroke and efficacy of antithrombotic therapy in atrial fibrillation. Analysis of pooled data from five randomized controlled trials. Arch Intern Med 1994;154:1449-57.

16 SPAF III Investigators. Transesophageal echocardiographic correlates of thromboembolism in high-risk patients with nonvalvular atrial fibrillation. The Stroke Prevention in Atrial Fibrilation Investigators Committee on Echocardiography. Ann Intern Med 1998; 128:639-47

- This important study from the SPAF III investigatiors highlights the role of TOE risk factor stratification in atrial fibrillation.

17 Leung DY, Davidson PM, Cranney GB, et al. Thromboembolic risks of left atrial thrombus detected by transesophageal echocardiogram. Am J Cardio 1997;79:626-9

18 Wyse DG, Waldo AL, DiMarco JP, et al. A comparison of rate control and rhythm control in patients with atrial fibrillation. N Engl J Med 2002;347:1825-33.

19 Klein AL, Grimm RA, Murray RD, et al. Use of transesophageal echocardiography to guide cardioversion in patients with atrial fibrillation. N Engl J Med 2001;344:1411-20.

- This landmark study was the first randomised, controlled comparison of TOE guided cardioversion versus a conventional approach.

20 Marrouche NF, Martin DO, Wazni O, et al. Phased-array intracardiac echocardiography monitoring during pulmonary vein isolation in patients with atrial fibrillation: impact on outcome and complications. Circulation 2003;107:2710-6.

21 Gillinov AM, McCarthy PM. Intraoperative bipolar radiofrequency clamp for intraoperative ablation of atrial fibrillation. Ann Thorac Surg 2002:74:2165-8.

22 Nakai T, Lesh MD, Gerstenfeld EP, et al. Percutaneous left atrial appendage occlusion (PLAATO) for preventing cardioembolism: first experience in canine model. Circulation 2002; 105:2217-22.

23 Sievert $\mathrm{H}$, Lesh MD, Trepels $\mathrm{T}$, et al. Percutaneous left atrial appendage transcatheter occlusion to prevent stroke in high-risk patients with atrial fibrillation: early clinical experience. Circulation 2002; 105:1887-9. 